\title{
Biomarqueurs aux phases précoces de développement dans la maladie d'Alzheimer
}

\author{
Régis Bordet ${ }^{1}$, Jean-François Dartigues ${ }^{2}$, Bruno Dubois ${ }^{3}$ Jean-Marie Goehrs ${ }^{4}$, Laura Vernoux ${ }^{5}$, Franck Semah ${ }^{6}$, \\ Florence Pasquier ${ }^{7}$, Claude Bidaut-Mazel ${ }^{8}$ et les participants à la Table Ronde $n^{\circ} 3$ de Giens XXV $V^{\star}$ \\ 1 EA 1046, Faculté de Médecine, Université de Lille II, Département Universitaire de Pharmacologie, Lille, France \\ 2 ISPED, Inserm U897, Université Victor Segalen Bordeaux 2, Bordeaux, France \\ 3 Fédération des Maladies du Système Nerveux, Hôpital de la Pitié-Salpétrière, Paris, France \\ 4 Faculté de Médecine Pierre et Marie Curie, Paris, France \\ 5 Innogenetics, Les Ulis, France \\ 6 Service de Médecine Nucléaire et Imagerie Fonctionnelle, CHRU Lille, France \\ 7 Pôle de Neurologie, Hôpital Salengro, CHRU Lille, France \\ 8 GlaxoSmithKline, Marly le Roi, France
}

Texte reçu le 4 mars 2010 ; accepté le 25 mai 2010

\begin{abstract}
Mots clés :
Alzheimer ;

développement clinique ; biomarqueur ;

imagerie

Résumé - Le développement clinique de médicaments disease-modifiers dans la maladie d'Alzheimer se heurte à des difficultés méthodologiques dont témoignent plusieurs échecs récents de médicaments en phase III. Compte-tenu des enjeux financiers inhérents au passage en phase III et du risque d'investir en pure perte de l'énergie pour l'évaluation d'un mauvais candidat-médicament, la question cruciale reste la décision de go/no go entre la phase II et la phase III dont l'objectif est certes de sélectionner une molécule susceptible d'être efficace en phase III mais plus encore d'écarter d'un développement ultérieur les candidats aux effets insuffisants. Aucun consensus n'existe aujourd'hui sur la meilleure conception possible des études de phase II pour tenter d'éclairer au mieux la décision de go/nogo. Les difficultés de choix de la meilleure conception d'étude concernent tout aussi bien la population-cible, les critères de jugement en particulier le recours à des biomarqueurs, le plan expérimental ou la durée des études. L'objet de la Table Ronde (TR) a été de rassembler les points de vue d'experts français issus du monde académique, industriel ou réglementaire afin d'arriver à une proposition consensuelle sur la meilleure conception possible que devraient utiliser les études de phase II dans la maladie d'Alzheimer.
\end{abstract}

\section{Maladie d'Alzheimer : phase prodromale et démence}

Les membres de la TR, en complément des résultats de celle de l'an dernier, ont réaffirmé la nécessité de distinguer la maladie d'Alzheimer dans son ensemble et la démence qui ne constitue qu'une étape évolutive de la maladie. Les membres de la TR ont bien repris à leur compte la notion de phase prodromale ou pré-démentielle de la maladie qui devrait être la cible des médicaments aujourd'hui en cours d'évaluation bien que l'observation des protocoles d'études déclarés sur www.clinical-trial.gov ont encore souvent des critères d'inclusion de maladie d'Alzhei-

\footnotetext{
* Pour la liste des participants, voir en fin d'article.
}

mer au stade de la démence, même si celle-ci est au stade léger à modéré. La discussion a évidemment alors pris en compte la notion de population sélectionnée qui peut conduire les autorités réglementaires à restreindre l'indication et le remboursement à ces seules populations-cibles. Les membres de la TR ont toutefois jugé qu'il était préférable d'avoir un médicament efficace sur une population restreinte qu' aucun médicament efficace et enregistré.

\section{Disease-modifiers : cours évolutif et effet physiopathologique}

Les membres de la TR ont discuté de la notion même de disease-modifiers considérant qu'un tel candidat-médicament doit 
être capable : (i) de modifier durablement le cours évolutif de la maladie; (ii) d'avoir un effet sur la cascade physiopathologique de la maladie. La différence entre un effet symptomatique et un effet disease-modifier réside, au plan théorique, dans le fait que la courbe d'évolution est durablement changée par le diseasemodifier même s'il a été arrêté alors que le changement d'évolution disparaît à l'arrêt du traitement purement symptomatique. Cependant la discussion est restée ouverte sur deux points. Le premier point concerne la nature de l'effet physiopathologique, avec l'interrogation de savoir si seule une action sur des mécanismes spécifiques de la maladie (cascade amyloïde, dégénérescence neurofibrillaire) doit être considérée ou si des actions moins spécifiques (inflammation, stress oxydant, fonctions vasculaires et métaboliques...) peuvent également être prises en compte dans l'effet disease-modifier. Le deuxième point concerne la stratégie à adopter vis-à-vis de la démonstration d'un effet physiopathologique. Celle-ci doit elle être concomitante de la démonstration de l'effet sur le cours évolutif par la mise en évidence d'un impact sur un biomarqueur de la cascade physiopathologique ou doit elle venir en appui secondaire de la démonstration que le candidatmédicament modifie avant tout l'évolution de la maladie, que cette évolution soit évaluée cliniquement ou par un biomarqueur non spécifique d'évolutivité. Même si les guidelines de l'European Medicine Agency (EMA) ${ }^{[1]}$ privilégient plutôt la première solution, il a paru raisonnable de prendre en compte le stade de développement du médicament et de considérer la démonstration d'un mécanisme physiopathologique comme une deuxième étape de la démonstration de l'effet thérapeutique et non comme un prérequis, d'autant que le mécanisme réel d'action d'un médicament est parfois différent de celui pour lequel il a été initialement développé. La valeur du biomarqueur comme outil décisionnel doit par ailleurs être périodiquement réévaluée à la lumière de tous les travaux disponibles comme l'a bien montré l'historique dans d'autres domaines thérapeutiques (densité minérale osseuse pour l'ostéoporose, extra systoles ventriculaires et prévention de l'infarctus du myocarde, etc.). ${ }^{[2]}$

\section{Quels biomarqueurs pour la maladie d'Alzheimer}

En raison des difficultés à démontrer l'efficacité des candidats-médicaments à l'aide des seuls outils cliniques et cognitifs, la nécessité d'un recours à des biomarqueurs s'est imposée depuis quelques années. Le biomarqueur est par définition un paramètre qui peut être mesuré de manière objective et sert d'indicateur d'un processus biologique normal, d'un processus pathologique ou de réponse pharmacologique à une intervention thérapeutique. Il peut constituer un critère de jugement intermédiaire (surrogate endpoint) lorsqu'il peut être substitué au critère de jugement clinique. Au-delà des échelles cognitives et psychocomportementales qui sont elles-mêmes des biomarqueurs de l'état clinique, des biomarqueurs génétiques, biologiques, neurophysiologiques ou d'imagerie ont été proposés au cours de la maladie d'Alzheimer. Certains sont en lien avec la cascade physiopathologique de la maladie d'Alzheimer, qu'ils soient des index de mécanismes spécifiques (cascade amyloïde ou phosphorylation anormale de la protéine tau) ou non spécifiques. D'autres sont des marqueurs de la souffrance cérébrale indépendamment des mécanismes physiopathologiques sous-jacents.

L'identification des biomarqueurs génétiques est la conséquence des multiples travaux d'épidémiologie génétique menés tant sur les formes familiales que sporadiques de maladie d'Alzheimer. Le marqueur le plus connu est l'allèle épsilon 4 du gène de l'apolipoprotéine E, qui constitue à la fois un index de susceptibilité à développer la maladie d'Alzheimer et un outil de stratification en termes de réponse thérapeutique. Les nombreux gènes associés à un risque accru de maladie d'Alzheimer et récemment identifiés sont autant de marqueurs possibles à intégrer au développement de nouveaux médicaments au cours de la maladie d'Alzheimer, car potentiellement associés à des réponses pharmacologiques différentes. Mais, si ils peuvent être utiles pour mieux définir la population cible, ils ne peuvent l'être pour évaluer l'efficacité thérapeutique.

Les progrès concernant les biomarqueurs biologiques ont été considérables au cours des cinq dernières années grâce à la meilleure caractérisation des voies physiopathologiques et aux résultats d'études clinico-biologiques d'envergure. Ces biomarqueurs biologiques reposent essentiellement sur le dosage dans le liquide céphalo-rachidien (LCR) de paramètres des cascades spécifiques de la maladie : voie amyloïde (Abeta 40, Abeta 42, rapport Abeta42/Abeta40, et d'autres formes tronquées d'Abeta); voie de la protéine tau (tau et phospho-tau). Ces marqueurs dosés dans le liquide céphalo-rachidien sont maintenant validés dans le diagnostic, en particulier précoce, de la maladie d'Alzheimer qui conduit à une harmonisation des procédures et des index entre les laboratoires, afin de standardiser ces dosages et de définir des algorithmes décisionnels. Si ces biomarqueurs du LCR sont des marqueurs d'état de la maladie permettant un diagnostic positif, il manque en revanche la démonstration du lien évolutif entre la progression de la maladie et des changements quantitatifs de ces biomarqueurs, excepté au stade léger de la maladie où la progression dans la démence de type Alzheimer est corrélée aux concentrations des biomarqueurs du LCR. ${ }^{[3]}$ Cette absence de démonstration est une limite à l'utilisation du dosage dans le LCR en tant que critère pharmacodynamique et a fortiori en tant que critère de substitution. Les limitations de la ponction lombaire expliquent le développement en cours des dosages plasmatiques 
des mêmes marqueurs spécifiques de la maladie d'Alzheimer. En dehors des marqueurs des voies spécifiques de la maladie d'Alzheimer, d'autres marqueurs peuvent également cibler des voies non spécifiques (inflammation, stress oxydant, marqueurs endothéliaux) qui pourraient également constituer à terme des indicateurs d'une action physiopathologique au cours de l'évaluation de candidats disease-modifiers ciblant des voies non spécifiques de la maladie.

Les biomarqueurs d'imagerie se développent de manière régulière tant à cause des progrès techniques et méthodologiques que grâce à une meilleure connaissance de la physiopathologie. ${ }^{[4,5]}$ L'imagerie repose sur deux types d'approches : (i) l'imagerie par résonance magnétique; (ii) l'imagerie nucléaire par Positron Emission Tomography (PET) ou Single Photonic Computerized Tomography (SPECT). On distingue deux types de biomarqueurs en imagerie : (i) les marqueurs indépendants de la physiopathologie qui authentifient la topographie du processus lésionnel neuronal [Imagerie par Résonnance Magnétique (IRM) avec analyse volumétrique, PET-fluorodeoxyglucose (PET-FDG), SPECT] pour lesquels l'Alzheimer Disease Neuro Imaging Initiative (ADNI I) a mis en évidence que l'IRM structurelle et le FDGPET ont montré une puissance statistiquement plus importante que les mesures cliniques et cognitives pour détecter un changement chez tous les sujets ; ${ }^{[6]}$ (ii) les marqueurs de processus physiopathologiques spécifiques (plaques amyloïdes, dégénérescence neurofibrillaire) ou non spécifique (activation microgliale). Concernant l'imagerie métabolique des processus physiopathologiques, des radiotraceurs permettent d'authentifier dans le cerveau des lésions amyloïdes (marqueurs de Pittsburgh : PIB, AV45...), la présence de dégénérescence neurofibrillaire (FDDNP) ou d'une activation microgliale (PK1195). Ces marqueurs d'imagerie sont d'abord une aide au diagnostic puisque en cas de déclin cognitif modéré de type amnésique, il est possible de poser le diagnostic de maladie d'Alzheimer probable en cas d'existence d'une atrophie hippocampique en IRM ou de présence de plaques amyloïdes en PET. D'un point de vue pharmacodynamique, une association a été mise en évidence entre le déclin cognitif et la progression des lésions en IRM de structure, en particulier l'atrophie globale ou l'atrophie hippocampique. En revanche, l'importance de plaques amyloïdes ne semble pas être différente en fonction du stade évolutif de la maladie, conduisant à considérer le PETamyloïde comme un marqueur d'état et non un marqueur d'évolutivité. La place du PET visualisant la dégénérescence neurofibrillaire et l'activation microgliale est encore du domaine de la recherche fondamentale.

Même si les données bibliographiques sont moins abondantes les concernant, les biomarqueurs neurophysiologiques sont loin d'être dénués d'intérêt. Ils permettent d'identifier des dysfonctions de l'activité cérébrale, de manière objective et reproductible. Certaines anomalies de la synchronisation en éléctro-encéphalogramme (EEG) peuvent être associées à un risque d'évolution vers une maladie d'Alzheimer avérée en cas de déclin cognitif léger. Ces anomalies électrophysiologiques ont pu être corrélées à l'importance du déclin cognitif même si l'association entre progression de la maladie et anomalies électrophysiologiques restent à démontrer.

\section{Essai de phase II : les différents aspects du protocole}

L'essai de phase II a un double objectif que recouvrent la preuve de concept, c'est-à-dire la démonstration que la cible choisie est pertinente, et l'étude dose-réponse en vue du choix de la posologie à tester en phase III. À partir de la définition de ces objectifs généraux, la répétition des échecs de plusieurs médicaments en cours de développement dans la maladie d'Alzheimer conduit cependant à affiner la conception des essais de phase II en termes de population-cible, de critères de jugement, le plan expérimental ou la durée des études. Deux éléments supplémentaires doivent également être pris en compte à ce stade : (i) la mise en évidence d'un impact sur la physiopathologie de la maladie à définir en fonction du mécanisme supposé du candidatmédicament; (ii) l'obtention rapide de données sur la sécurité d'emploi, les mécanismes d'action utilisés accroissant le risque d'effets indésirables graves comme l'a montré la première tentative d'immunothérapie anti-beta. Si l'objectif final du développement d'un disease-modifier dans la maladie d'Alzheimer reste bien le ralentissement de la pente d'évolution du déclin cognitif, il est nécessaire de mieux concevoir les études afin de distinguer les candidats-médicaments qui n'atteignent pas un niveau de preuve suffisant pour poursuivre leur développement de ceux dont la démonstration d'effet semble suffisamment solide pour autoriser une décision de passage en phase III. En terme de population-cible, il paraît encore plus crucial en phase II, d'évaluer les candidatsmédicament sur des patients qui sont les plus à même de tirer bénéfice d'un disease-modifier, c'est-à-dire les patients à un stade peu évolué de la maladie plutôt que chez des patients déjà au stade de démence chez lesquels le processus physiopathologique est déjà peut-être trop évolué pour pouvoir être modulé pharmacologiquement. À ce stade, il est probablement nécessaire d'enrichir la population cible en tenant compte du polymorphisme génétique ou de la présence de lésions vasculaires voire de stratifier afin d'identifier des populations répondeuses/non répondeuses pour permettre d'accroitre secondairement les chances de succès de la phase III. En matière de critère de jugement, il paraît peu opérant à ce stade de se focaliser sur des critères cliniques et cognitifs mais plutôt d'évaluer les candidat-médicaments à l'aide de méthodes qui permettent de mieux objectiver l'impact sur la progression de 
la maladie en s'affranchissant du risque de mettre en évidence un effet purement symptomatique qui est toujours possible avec des critères cliniques. En effet, il n'est pas légitime d'envisager en phase II le recours à des dessins d'étude de type delayed start qui, s'ils permettent de distinguer effet symptomatique et effet diseasemodifier, sont probablement peu adaptés à ce stade de développement des médicaments. En effet, en phase II, il semble raisonnable d'utiliser un plan classique en groupes parallèles et contre placebo. Si pour des traitements symptomatiques des durées d'essai courtes (3 à 6 mois) permettent de mettre en évidence un effet significatif, il semble raisonnable, compte-tenu de la pente de déclin [perte d'environ 3 points au mini mental status (MMS) par an], de prévoir une durée d'un an pour les phases II afin d'avoir une chance de pouvoir mettre en évidence un changement de pente. Ce qui pose la question de «l'add-on thérapie», car il est illusoire de pouvoir maintenir pendant un an un patient atteint de maladie d'Alzheimer au stade prodromal ou au stade léger sans aucun traitement.

Au stade de la phase II, l'utilisation de biomarqueurs doit être envisagée à toutes les étapes de conception des études : diagnostic, enrichissement, stratification, évaluation, mécanisme, sécurité. Si des paramètres cliniques sont indispensables, ils ne sont peut-être pas ceux à mettre au premier plan, au profit des biomarqueurs biologiques, génétiques, d'imagerie ou neurophysiologiques. Cependant, tous ces biomarqueurs ne sont peut-être pas pertinents pour tous les aspects de l'étude de phase II, nécessitant une analyse critique de l'intérêt de ces biomarqueurs pour tous les aspects de la phase II en les sélectionnant et en les hiérarchisant.

\section{Quels biomarqueurs et pour quels paramètres?}

À l'issue de la discussion du panel d'experts, un poids relatif a été attribué, par consensus, à chaque type de biomarqueurs pour les différents aspects de la phase II (tableau I). Dans le domaine du diagnostic, en supplément de l'existence de symptômes cognitifs caractérisés sous forme d'un syndrome hippocampique amnésique, les biomarqueurs d'une voie physiopathologique spécifique de la maladie d'Alzheimer apparaissent les plus pertinents qu'ils soient des marqueurs biologiques, comme le dosage d'Abeta $40,{ }^{[7]}$ de tau et phospho-tau dans le liquide céphalorachidien ou des marqueurs d'imagerie, comme l'identification de plaques amyloïdes en PET à l'aide de radio-traceurs spécifiques. En deuxième niveau, les biomarqueurs de topographie lésionnelle (IRM volumétrique, PET-FDG) peuvent étayer l'existence du processus dégénératif aux phases précoces de la maladie aidant ainsi au diagnostic positif nécessaire à l'inclusion dans les essais. En matière d'enrichissement, un des objectifs est de pouvoir affiner le stade évolutif de la maladie à l'aide de biomarqueurs se modifiant en fonction de la progression de la maladie (LCR-p-tau, LCR-ttau, ${ }^{[7]}$ ou ratio Abeta42/p-tau181, ${ }^{[8]}$ atrophie en IRM, altération du métabolisme en PET-FDG) ou de marqueurs susceptible d'influencer l'évolutivité de la maladie ou la réponse au traitement (PET-FDG qui peut être un marqueur d'efficacité). Fondée sur cette possibilité d'enrichir le profil de la population étudiée dans l'essai, une stratification est probablement nécessaire, s'appuyant sur des critères permettant de distinguer des populations ayant des niveaux différents de réponse au traitement évalué. Par analogie avec le traitement symptomatique, une stratification en fonction du polymorphisme génétique de l'apolipoprotéine E peut être proposée même si, finalement à l'avenir, d'autres polymorphismes se révéleront peut-être plus intéressants. Une deuxième approche est de stratifier sur l'importance de la charge lésionnelle, qu'elle soit spécifique (PET-amyloïde ou LCR-tau, p-tau181 ou ratios élevés tau(s)/Abeta42) ${ }^{[3,8]}$ ou non spécifique (IRM volumétrique, PETFDG), en partant de l'hypothèse que les patients les moins évolués au plan lésionnel pourraient avoir une modification plus importante de leur pente évolutive. Au plan des critères d'évaluation, le panel d'experts n'a pas considéré que les biomarqueurs biologiques étaient à ce stade les plus pertinents compte-tenu de l'impossibilité de répéter les ponctions lombaires. Le développement et la validation de kits de dosages plasmatiques conduiraient à revoir ce jugement. Le PET-FDG et l'IRM volumétrique, témoins d'une lésion évolutive sans qu'elle soit typée au plan physiopathologique, ont été jugés comme les biomarqueurs les plus pertinents en tant que critères de jugement. En l'absence d'évolutivité démontrée, le PET-amyloïde n'a été retenu que comme biomarqueur de deuxième niveau. Les critères cliniques ont également été jugés comme des index d'efficacité de deuxième niveau. Par référence aux méningo-encéphalites induites par la première approche en immunothérapie testée, il a été jugé que l'IRM morphologique pouvait également apporter des indications en matière de sécurité. Les experts ont également jugé que des nouveaux biomarqueurs pouvant être utilisés comme critères de jugement devait être développés. De ce point de vue, l'IRM fonctionnelle et les marqueurs neurophysiologiques peuvent être une piste qui sera étudiée dans les années à venir dans le cadre du projet Pharmacog, soutenu par l'Innovative Medicine Initiative [European Union (EU) \& l'European Federation of Pharmaceutical Industries and Association (EFPIA)]. En matière de mise en évidence d'un mécanisme d'action, il est apparu clairement aux experts que, pour l'heure, seuls les biomarqueurs biologiques ou d'imagerie ciblant une cascade physiopathologique spécifique de la maladie d'Alzheimer étaient pertinents, à la réserve que cette étude n'est valable que pour les approches pharmacologiques modulant cette cascade particulière. 
Tableau I. Poids relatif attribué aux différents bio-marqueurs par la Table Ronde ${ }^{\circ} 3$ de Giens. (d'après les conclusions de la Task Force d’Oxford 2009). [10]

\begin{tabular}{|c|c|c|c|c|c|c|}
\hline \multicolumn{2}{|c|}{ Type de Biomarqueur } & Diagnostic & $\begin{array}{c}\text { Enrichissement } \\
\text { de population }\end{array}$ & Stratification & $\begin{array}{c}\text { Evaluation } \\
\text { de l'effet }\end{array}$ & $\begin{array}{c}\text { Mécanisme } \\
\text { d'action }\end{array}$ \\
\hline \multirow{5}{*}{ Bio-LCR } & $\mathrm{A} \beta(40+42)$ & +++ & $?$ & + & + & +++ \\
\hline & TotalTau & ++ & $?$ & - & + & ++ \\
\hline & Phos Tau & ++ & ++ & + & + & +++ \\
\hline & IATI & +++ & $?$ & $?$ & + & +++ \\
\hline & BACE 1 & + & & - & $?$ & +++ \\
\hline Bio-Plasma & $\mathrm{A} \beta(40+42)$ & - & + & - & + & ++ \\
\hline \multirow{2}{*}{ Bio-Autres } & Inflammatoires & - & - & - & - & + \\
\hline & Vasculaires & - & - & - & - & + \\
\hline \multirow{2}{*}{ Génétiques } & Statut ApoE & - & +++ & +++ & - & + \\
\hline & Kits et sets de gènes & - & - & - & - & + \\
\hline \multirow{5}{*}{ Imagerie } & PET (charge amyloïde) & +++ & +++ & ++ & ++ & +++ \\
\hline & FDG-PET & ++ & ++ & ++ & +++ & + \\
\hline & fIRM & - & - & - & + & - \\
\hline & SPECT & + & - & - & + & - \\
\hline & IRM structurelle & ++ & + & ++ & $+++(+$ tolérance $)$ & - \\
\hline Neurophysiologie & EEG/ Potentiels évoqués & - & + & + & $?$ & - \\
\hline Cognitif & Sd amnésique hippocampique & +++ & +++ & + & ++ & - \\
\hline
\end{tabular}

A $\beta$ : peptide amyloïde ; BACE 1 : Beta site APP Cleaving Enzyme 1 ; EEG : électro-encéphalogramme ; IATI : Innotext Amyloid Tau Index; IRM : Imagerie par Résonnance Magnétique (fIRM : Imagerie par Résonnance Magnétique fonctionnelle); Sd : syndrome; SPECT : Single Photonic Computerized Tomography.

Tableau II. Choix des biomarqueurs à la Phase II du développement clinique.

\begin{tabular}{lll}
\hline Biomarqueur diagnostique & Biomarqueur d'évaluation & $\begin{array}{l}\text { Biomarqueur du mécanisme } \\
\text { (molécule dépendant) }\end{array}$ \\
\hline 1- IRM + LCR & $1-$ FDG & 1- LCR + plasma \\
2- IRM + imagerie amyloïde & $2-$ IRMv & 2- imagerie amylö̈de \\
3- IRM + FDG & & \\
\hline
\end{tabular}

IRM : Imagerie par Résonnance Magnétique ; IRMv : Imagerie par Résonnance Magnétique volumétrique ; LCR : Liquide Céphalo-Rachidien ; FDG : Fluorodeoxyglucose.

\section{Choix des biomarqueurs à la phase II et conception d'étude}

Une fois le travail de sélection des biomarqueurs effectué, les experts du panel se sont attachés à définir un design d'étude de phase II à 3 niveaux principaux : (i) critères de sélection ; (ii) critères de jugement; (iii) critères d'effet physiopathologique (tableau II). En matière de critères de sélection, l'objectif est d'inclure des patients au stade prodromal au sens des critères de Dubois ${ }^{[9]}$ qui constitue la population la plus à même de bénéficier d'un traitement disease-modifier. Si le traitement est spécifique du processus physiopathologique de la maladie d'Alzheimer considéré actuellement le plus probable, la cascade amylö̈de, le critère d'inclusion doit être un syndrome amnésique hippocampique associé en première intention à une IRM montrant une atrophie hippocampique et une analyse du liquide céphalo-rachidien montrant une baisse de la concentration Abeta42 ou du ratio Abeta42/40 et une augmentation de la concentration de phospho-tau. L'alternative au plan paraclinique peut être : (i) une IRM avec atrophie hippocampique et la mise en évidence de plaques amyloïdes en PET; (ii) une IRM avec atrophie hippocampique et un hypométabolisme en PET-FDG. En matière de critères de jugement, un consensus s'est dégagé pour considérer que le critère de jugement principal devait être une modification de la progression de lésions non spécifiques témoins de la perte neuronale («marqueurs topographiques »), en raison des données montrant une association entre le déclin cognitif et la progression des lésions visualisées en imagerie morphologique ou métabolique. En première intention, il est apparu que le PET-FDG pouvait être le critère le plus sensible à une action pharmacologique. L'IRM volumétrique a été considérée comme l'alternative en termes de critère de jugement. Le critère de jugement secondaire doit être un critère clinique, plus particulièrement l'Alzheimer Disease Assessment Scale-Cognitive (ADAS-cog) qui reste la référence. Le choix des 
Tableau III. Algorythme de décision de passage en phase III (« go/no go »).

\begin{tabular}{lllll}
\hline FDG & + & - & + & - \\
ADAS-Cog & - & + & + & - \\
Go/NoGo & Go & Go & Go & NoGo \\
\hline
\end{tabular}

ADAS-Cog : Alzheimer Disease Assessment Scale-Cognitive; FDG : Fluorodeoxyglucose.

critères physiopathologiques reste un choix par défaut dans la mesure où les biomarqueurs existants et validés restent les marqueurs de la cascade amyloïde. Si ces marqueurs sont pertinents pour des agents pharmacologiques modulant la cascade amyloïde, ils restent totalement inopérants pour toutes les autres approches. $\mathrm{Ce}$ nouveau concept d'étude n'est pas en contradiction avec les guidelines de l'EMA ${ }^{[1]}$ qui sont évolutifs et qui reconnaissent déjà, dans la maladie d'Alzheimer, les travaux effectués sur les biomarqueurs même si leur validation reste encore nécessaire. L'établissement d'un effet clinique sur le déclin cognitif et fonctionnel en phase III est déjà accepté comme un effet sur la progression de la maladie et qui, soutenu par un effet sur un biomarqueur biologique et/ou d'imagerie, pourrait aboutir à une démonstration d'effet sur le processus pathologique (disease-modifier).

\section{Algorithme de décision de passage en phase III (go/no go)}

Au-delà de la conception de l'étude, les experts se sont également interrogés sur l'attitude à adopter vis-à-vis de la décision de poursuivre le développement en phase III, en fonction des résultats obtenus pour les critères primaire et secondaires choisis (tableau III). Dans une situation idéale, le candidat-médicament se révèle avoir un effet bénéfique à la fois sur la progression des lésions en imagerie (PET-FDG, ou à défaut atrophie en IRM) et du déclin cognitif. La décision de passage en phase III ne se discute pas. Si le candidat-médicament induit un effet bénéfique sur le déclin cognitif, sans effet démontré sur les marqueurs d'imagerie, la décision semble facile à prendre en raison de l'existence d'un effet clinique, l'absence d'impact sur un critère objectif de lésion cérébrale, expose au risque de ne démontrer qu'un effet symptomatique avec des dessins d'étude de phase III plus adaptés à la démonstration d'un effet disease-modifier. L'existence d'un effet sur des critères objectifs d'imagerie en l'absence d'effet clinique peut paraître, en première intention, plus risqué. Mais à ce stade, la sensibilité des critères cliniques peut être moins importante que celle des critères d'imagerie justifiant la prise de risque de poursuite en phase III. En revanche, il est clair qu'en l'absence d'effet démontré tant au plan clinique qu'au plan de l'imagerie, le groupe d'experts a estimé que le développement devrait être stoppé à ce stade afin d'éviter une déperdition de moyens et d'énergie pour l'étude d'un agent qui a peu de chance d'aboutir.

\section{Conclusion}

Afin de minimiser l'échec du développement clinique des molécules visant la maladie d'Alzheimer, l'objectif est probablement, dans les années à venir de limiter le risque d'échec en phase III, en améliorant la prise de décision de go/no go à l'issue de la phase II. Cette décision ne peut plus se faire uniquement sur des critères de jugement clinique et chez des patients mal sélectionnés et présentant une maladie déjà évoluée. Un consensus d'experts français a conclu à la nécessité d'étudier en phase II des patients présentant une forme prodromale de la maladie d'Alzheimer diagnostiqués à la fois sur des critères cliniques de syndrome amnésique hippocampique et sur des biomarqueurs biologiques et d'imagerie. La démonstration, à ce stade de développement, d'un effet bénéfique sur la progression des marqueurs lésionnels non spécifiques en imagerie et/ou sur le déclin cognitif doit conforter la prise de décision de poursuite du développement, en sachant que la situation idéale est la démonstration à la fois d'un effet lésionnel et clinique. En revanche, le groupe d'experts a considéré qu'il n'y avait pas lieu de poursuivre en phase III le développement d'un candidat-médicament n'ayant ni effet clinique ni effet sur la progression des lésions. Cette réflexion devrait aider les industriels dans la conception des études de phase II et dans leur prise de décision de poursuite ou non du développement en fonction des résultats. Ce développement optimisé constitue une base de réflexion pour optimiser le développement des nouvelles molécules dans la maladie d'Alzheimer, en évitant la déperdition financière et d'énergie dont le risque est d'aboutir, faute de résultat, à désengagement de la recherche industrielle dans ce champ, à l'instar de ce qui s'est passé dans la pathologie vasculaire cérébrale.

Participants. Zeina Antoun (Laboratoire GlaxoSmithKline), Olivier Arnaud (Inserm Transfert, Paris), Olivier Blin (CICUPCET, Marseille), Antoine Coquerel (CHU Côte de Nacre, Caen), Catherine Deguines (Afssaps, Saint-Denis), Philippe Derambure (CHRU, Lille), Patrice Dosquet (Haute Autorité de Santé, Saint-Denis La Plaine), Jean-Pierre Duffet (DHOS-OPRC, Antony), Sylvia Goni (Laboratoire Lundbeck), Philippe Gustovic (Wyeth Pharmaceuticals), Marie Lang (Ministère de la Santé, Paris), Marie-Laure Laroche (CRPV, CHU Dupuytren, Limoges), Antoine Pariente (Inserm U 657, Bordeaux), Jean-Jacques Pere (Laboratoire Novartis), Odile Regnier (Cnamts, Paris), Philippe Truffinet (Laboratoire Sanofi Aventis). 


\section{Références}

1. CHMP 2008. Guideline on medicinal products for the treatment of Alzheimer's disease and other dementias. http: //www . emea. europa.eu/ pdfs/human/ewp/ $\backslash 1 \backslash 055395$ en.pdf

2. Lathia CD, Amakye D, Dai W, et al. State of the art. The value, qualification, and regulatory use of surrogate end points in drug development. Clinical Pharmacology \& Therapeutics 2009; 86 (1): 32-43

3. Snider BJ, Fagan AM, Roe C, et al. Cerebrospinal fluid biomarkers and rate of cognitive decline in very mild dementia of the Alzheimer type. Arch Neurol 2009; 66(5): 638-45

4. Jack CR, Bernstein MA, Borowski BJ, et al. Update on the magnetic resonance imaging core of the Alzheimer's disease neuroimaging initiative. Alzheimer's \& Dementia 2010; 6(3): 212-20

5. Jagust WJ, Bandy D, Chen K, et al. The Alzheimer's disease neuroimaging initiative positron emission tomography core. Alzheimer's \& Dementia 2010; 6(3): 221-9

6. Walhovd KB, Fjell AM, Brewer J, et al. Combining MR imaging, positronemission tomography, and CSF biomarkers in the diagnosis and prognosis of Alzheimer disease. American Journal of Neuroradiology 2010; 31: $347-54$
7. Van der Vlies AE, Verwey NA, Bouwman FH, et al. CSF biomarkers in relationship to cognitive profiles in Alzheimer Disease SYMBOL. Neurology 2009; 72(12): 1056-61

8. Fagan AM, Mintun MA, Shah AR, et al. Cerebrospinal fluid tau and ptau181 increase with cortical amyloid deposition in cognitively normal individuals: implications for future clinical trials of AD. EMBO Molecular Medicine 2009; 1(8-9): 371-80

9. Dubois B, Feldman HH, Jacova C, et al. Research criteria for the diagnosis of Alzheimer's disease: revising the NINCDS-ADRDA criteria. Lancet 2007; 6: $734-46$

10. Vellas B, Andrieu S, Aisen P, et al. for the Oxford Task Force Group. Biomarkers for Alzheimer's disease therapeutic trials: The Oxford Task Force. À paraître

Correspondance et offprints : Claude Bidaut-Mazel, GlaxoSmithKline Direction Médicale, 100 route de Versailles, 78163 Marly le Roi Cedex, France.

E-mail : claude.m.bidaut-mazel@gsk.com ; claudebidaut-mazel@orange.fr 